\title{
Integrating sex- and tissue-specific regulation within a single Drosophila enhancer
}

\author{
Wenqian An and Pieter C. Wensink ${ }^{1}$ \\ Department of Biochemistry and The Rosenstiel Center, Brandeis University, Waltham, Massachusetts 02254-9110 USA
}

\begin{abstract}
We have investigated the integration of sex- and tissue-specific transcriptional regulation in Drosophila. A single copy of the o-r enhancer from yolk protein genes directs female- and fat body-specific transcription. It consists of four protein-binding sites: $\mathrm{dsXA}$, which binds male $\left(\mathrm{DSX}^{\mathrm{M}}\right)$ and female (DSX $\left.\mathrm{X}^{\mathrm{F}}\right)$ proteins encoded by the doublesex gene; aef1, which binds the AEF1 repressor; bzip1, which binds the DmC/EBP activator encoded by the slbo gene; and ref1, which binds an unknown activator. Multimeric and mutated binding sites were used in protein binding, germ-line transformation, and genetic experiments to examine the independent and combinatorial activities of the proteins and DNA sites. DSX $^{\mathrm{F}}$ activates from dsxA by sterically excluding AEF1 repressor from the aef1 site and synergistically activating transcription together with a protein at bzip1. Sex specificity in fat bodies arises from the opposite effect of $\mathrm{DSX}^{\mathrm{M}}$, which represses activity of the protein at bzip1. Tissue specificity is regulated by all four DNA sites. Separately, bzip1 and ref1 activate transcription in ovarian somatic cells and all nongonadal tissues, respectively, whereas together they activate only in fat bodies. The aef1 site represses ectopic transcription in ovaries and dsxA antirepresses this activity in fat bodies. Thus, in the organism, ref1 and bzip1 act combinatorially to direct the fundamental tissue specificity, aef1 and dsxA modulate this tissue specificity, and dsxA adds sex specificity.
\end{abstract}

[Key Words: Sexual differentiation; yolk protein genes; AEF1; doublesex]

Received October 19, 1994; revised version accepted December 14, 1994.

Studies indicate that the very large number of developmentally specific transcription patterns occurring in a higher organism is controlled by a much smaller number of transcriptional regulatory proteins (for review, see Struhl 1991). An explanation for this difference in numbers is that a narrow tissue specificity can be regulated by the combinatorial activity of several proteins that may be quite broadly distributed among tissues (e.g., Orkin 1990; De Simone and Cortese 1991; Weintraub et al. 1991; Lawrence 1992; Nelsen and Sen 1992; Small et al. 1992).

The yolk protein $(Y p)$ genes of Drosophila melanogaster provide a good opportunity to investigate molecular mechanisms that integrate a broad and well-characterized developmental specificity, sexual identity, with a narrow tissue specificity. We have recently identified a small enhancer, $\underline{\mathrm{o}-\mathrm{r}}$, that lies between the $Y p 1$ and $Y p 2$ genes and directs female- and fat body-specific transcription similar to that of the normal Yp genes (An and Wensink 1995). The o-r enhancer consists of four proteinbinding sites organized into two enhancer elements. One element, $\underline{0}$, contains three overlapping sites: dsxA, aef 1 , and bzipl which bind, respectively, the female (DSX ${ }^{\mathrm{F}}$ )

${ }^{1}$ Corresponding author. and male $\left(\mathrm{DSX}^{\mathrm{M}}\right)$ regulatory proteins from the doublesex $(d s x)$ gene, the AEF1 repressor, and the DmC/EBP transcriptional activator. The other element, $\underline{\mathbf{r}}$, is a single protein-binding site, refl, which binds an unidentified activator. Germ-line transformation studies showed that as is typical of enhancer elements, neither o nor $\underline{r}$ can activate transcription by themselves, but together in single copy they direct transcription only in female fat bodies (An and Wensink 1995). With the exception of aefl, all of the binding sites in the complete $\underline{0-r}$ enhancer are necessary for this transcription pattern.

The proteins that bind to $\underline{0-r}$ have all been implicated in either the tissue- or sex-specific regulation of transcription. DmC/EBP, a member of the bZIP family of regulatory proteins, is encoded by the slow border cells (slbo) gene of Drosophila (Montell et al. 1992; Rørth and Montell 1992). It is a homolog to mammalian C/EBP $\alpha$, which appears to have a general role in regulating terminal cell type differentiation in mammals (Birkenmeier et al. 1989). Both C/EBP $\alpha$ and DmC/EBP activate from Drosophila enhancers in transfected cultured cells (Falb and Maniatis 1992a; T. Maniatis, pers. comm.). Similarly, the Drosophila AEF1 protein represses through an alcohol dehydrogenase (Adh) enhancer in transfected cultured cells (Falb and Maniatis 1992a,b). The final two proteins known to bind $\underline{0-r}$ are the male and female spe- 
cific dsx proteins. These proteins act directly on a larger $Y p$ enhancer that includes the $\operatorname{dsxA}$ site as well as two other dsx binding sites, causing transcriptional repression in males (DSX ${ }^{\mathrm{M}}$ ) and activation in females (DSX ${ }^{\mathrm{F}}$ ) (Coschigano and Wensink 1993; An and Wensink 1995). These two proteins are translated from alternative splice products of the primary dsx transcript (Burtis and Baker 1989|. Sex-specific alternative splicing is regulated by the well-studied somatic sex differentiation pathway of Drosophila (Burtis and Wolfner 1992; McKeown 1992; Burtis 1993). The two dsx proteins are identical over their first 397 amino acids, which include a DNA-binding domain (Erdman and Burtis 1993). In addition to this common sequence, $\mathrm{DSX}^{\mathrm{M}}$ has a 152-amino-acid carboxyl terminus that is completely different from the 30 -aminoacid carboxyl terminus of $\mathrm{DSX}^{\mathrm{F}}$.

We report molecular and genetic studies of the function of $\underline{0-r}$ binding sites and proteins. The observations outline individual activities and lead to the following model for the combinatorial protein interactions that integrate sex and tissue specificity in the normal o-r enhancer. Proteins acting at the bzipl and refl sites have narrow and broad tissue distributions, respectively, but when brought together by a single copy of the two sites, the proteins produce strongly synergistic activation of transcription only in fat bodies. Proteins acting at bzipl have the potential to activate transcription in other tissues, but this potential is repressed by the AEFl protein acting at the aefl site. The female sex specificity of this transcription is regulated only by dsx proteins acting at the dsXA site. DSX ${ }^{\mathrm{F}}$ activates in females through positive synergy with the protein at bzipl and through antirepression of AEF1 activity at the aefl site. In males, DSX $^{M}$ represses activity of the protein at bzipl. Overall, there are two levels of regulation. The first is synergistic tissue-specific activation by proteins binding to two well-separated sites. The second level is modification of this activation by positively and negatively regulating the activity of one of the first level sites. This modification is by the two dsx proteins regulating sex specificity and by AEF1 repressing ectopic transcription in a few non-fat body tissues.

\section{Results \\ A tetramer of the o enhancer element is sufficient to activate female-specific transcription in fat bodies}

The 29-bp enhancer element, $\mathrm{o}$, from the Drosophila Yp genes is composed entirely of three overlapping binding sites for different transcriptional regulatory proteins (Fig. 1). Like other enhancer elements (Ondek et al. 1988; Tjian and Maniatis 1994), a single copy of o is not an enhancer but does activate transcription when combined with a second enhancer element. Together with a single copy of the 11-bp $\leq$ element, o directs the sex-and fat body-specific expression characteristic of the Yp genes (Fig. 1; An and Wensink 1995).

To identify the independent regulatory properties of $\mathrm{o}$, we placed two and four copies of it upstream of a $\beta$-ga-

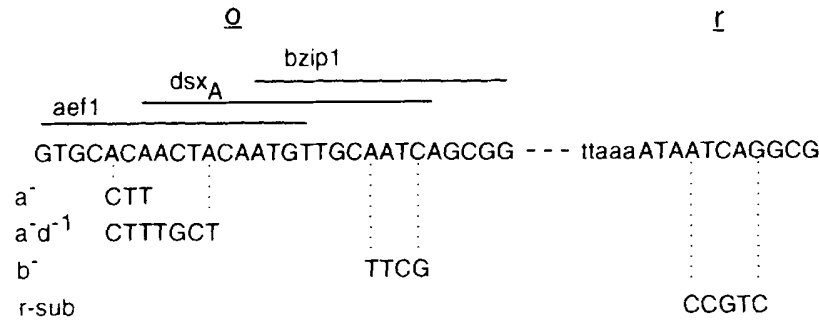

Figure 1. Wild-type and mutated $\underline{o}$ and $\underline{r}$. The wild-type nucleotide sequences for $\mathrm{o}(29 \mathrm{bp} ; l e f t)$ and $\mathbf{r}(11 \mathrm{bp}$, uppercase letters, right) are shown. The three DNase I footprints in $\underline{o}$ are indicated by solid lines (aef 1, dsxA, and bzip1). The mutations in $\underline{0}$ and $\underline{I}$ are shown below, aligned under the substituted wild-type sequences. These mutations reduce in vitro binding by AEF1 $>20$ fold $\left(\mathrm{a}^{-}\right)$and $>40$-fold $\left(\mathrm{a}^{-} \mathrm{d}^{-1}\right)$; DmC/EBP $($ BRLZ) $>50$-fold (b-) i DSX >25-fold $\left(\mathrm{a}^{-} \mathrm{d}^{-1}\right)$ (An and Wensink 1995). The $\underline{\mathrm{r}}$ substitution ( $\underline{\mathrm{r} \text {-sub }}$ ) completely abolishes $\underline{\mathrm{o}-\mathrm{r}}$ enhancer activity in vivo (An and Wensink 1995). The lowercase letters immediately upstream of $\underline{\underline{r}}$ show additional $Y p$ sequence tetramerized

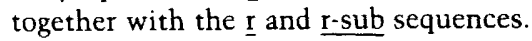

lactosidase reporter gene and introduced the product into the Drosophila germ line by P-element-mediated transformation. The specificity of the resulting expression is essentially the same with each multimer, but the dimer directs weak expression whereas the tetramer directs strong expression (Fig. 2a,b). Staining for $\beta$-galactosidase activity shows female-specific expression that occurs mainly in fat body tissue (Fig. 2a,b). Unlike the o-r enhancer, the o tetramer produces weak staining in two structures of ovarian egg chambers: the micropyle and, occasionally, the border cells (Fig. 2i). Weak and inconsistent staining also appears in the maxillary palpus but may be attributable to diffusion of stain from nearby and intensely staining fat bodies in the head.

All fat body-and sex-specific staining was directed by the regulatory protein-binding sites in o and not by other sequences in the reporter or spacer DNA. This was demonstrated by introducing 11-bp changes into $\underline{o}$ that reduce binding at least 20 -fold between o and the three regulatory proteins (Fig. 1, $\mathrm{a}^{-} \mathrm{d}^{-1} \mathrm{~b}^{-} ; \overline{A n}$ and Wensink 1995). A tetramer with these mutations produced no staining in fat bodies or head appendages [Fig. 2 , b vs. $f$ $\left(a^{-} d^{-1} b^{-}\right)_{4}$ vs. $\left.\left(a^{+} d^{+} b^{+}\right)_{4}\right]$. With this altered $o$, the only staining above background occurs in egg chambers, suggesting that either regulatory proteins acting at the three sites regulate differently in those egg chamber tissues or this expression is not attributable to o. We favor the former interpretation because the reporter gene alone does not express in egg chambers (Coschigano and Wensink 1993) and because of the individual activities of aefl and bzipl revealed by the experiments described below.

We conclude that tetramerized $\underline{o}$ is sufficient to activate transcription. Moreover, it retains the sex specificity and much of the tissue specificity of the entire $Y p$ genes in fat bodies. These results also show that the three transcription factor-binding sites in o contain information sufficient for these expression specificities. 

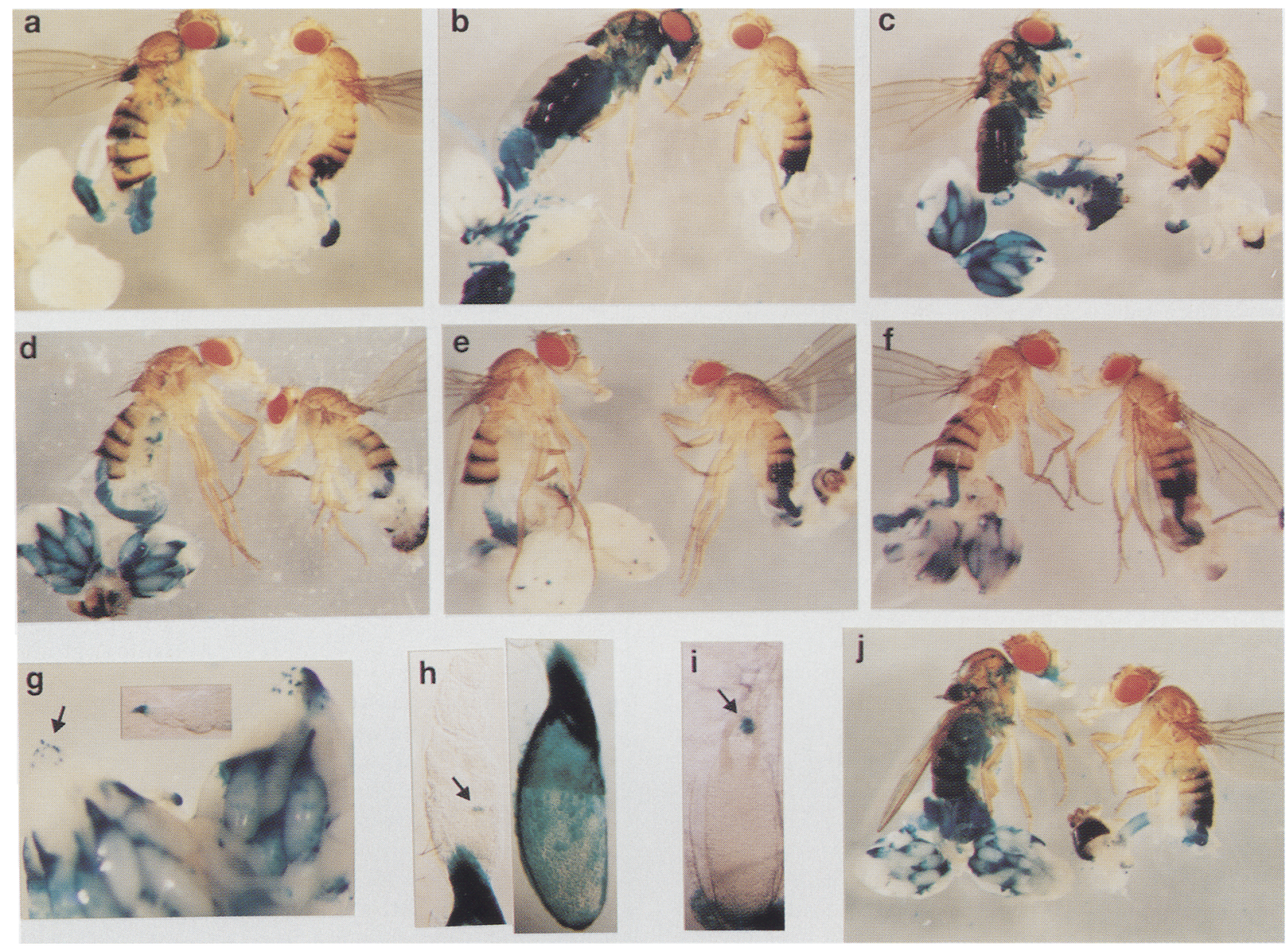

Figure 2. $\beta$-Galactosidase activity distribution in flies transgenic with o multimers. $(a-f)$ Flies with, respectively, $\left|a^{+} d^{+} b^{+}\right|_{2}$, $\left(a^{+} d^{+} b^{+}\right)_{4},\left(a^{-} d^{+} b^{+}\right)_{4},\left(a^{-} d^{-1} b^{+}\right)_{4},\left(a^{-} d^{+} b^{-}\right)_{4}$, and $\left(a^{-} d^{-1} b^{-}\right)_{4} \cdot(g)$ Ovaries from flies with $\left(a^{-} d^{+} b^{+}\right)_{4}$. The arrow points to the stained germarium, which is magnified further in the inset. (h) Egg chambers from flies with $\left(\mathrm{a}^{-} \mathrm{d}^{+} \mathrm{b}^{+}\right)_{4}$; (left), stage 10 egg with border cell staining (arrow); (right) stage 13 with uneven staining in main body follicle cells and strong staining in the dorsal appendage region, which includes the micropyle derived from border and centripetal follicle cells. (i) Stage 13-14 egg chamber from flies with $\left(a^{+} d^{+} b^{+}\right)_{4}$ showing a stained micropyle (arrow). The staining is restricted to the micropyle region. $(j)\left(\mathrm{a}^{-} \mathrm{d}^{+} \mathrm{b}^{+}\right)_{4}$ in flies carrying $d s x$ mutants; (left) one $d s x$ mutant allele; (right) two $d s x$ mutant alleles (note the strong intersexual phenotype of no ovaries or testes).

The aef1 site has no effect in fat bodies, but represses transcription in ovaries

We examined the regulatory specificities of individual protein-binding sites in o using germ-line transformation of o tetramers that have the site-selective mutations described in Figure 1. The 3-bp changes that reduce AEF1 binding $>20$-fold in vitro while having no effect on binding by the other two proteins also have no detectable effect on fat body transcription [Fig. 2, b vs. c $\left(a^{-} d^{+} b^{+}\right)_{4}$ vs. $\left.\left(a^{+} d^{+} b^{+}\right)_{4}\right)$. This result indicates that the bzipl and dsxA sites are sufficient for fat body specificity.

Although aef 1 has no effect on fat body expression, it represses ovarian expression, demonstrating that in the tetramer context, the bzipl and/or dsxA sites have additional transcriptional specificities [Fig. 2, b vs. c $\left(\left.a^{-} d^{+} b^{+}\right|_{4}\right.$ vs. $\left(\left.a^{+} d^{+} b^{+}\right|_{4}\right]$. Whereas the wild-type tetramer stains weakly in the oocyte micropyle and perhaps in the border cells of early egg chambers, the aef 1 mutation leads to strong and consistent staining of these regions. The mutation also gives staining in the tunica propria/terminal filament region of the germarium and, at later stages of egg chamber development, in several follicle cell types: stage 10, border cells; stage 12-14, intense staining in the posterior pole cells and the cells surrounding dorsal appendages, and uneven staining in the main body cells (Fig. 2c,g,h).

We conclude that the aefl site and, presumably, the AEFl repressor protein, have no effect on fat body transcription, but repress ovarian transcription directed by dsxA and/or bzipl. The lack of aefl repression in fat bodies contrasts with the repression in fat bodies observed for an AEFl-binding site in the adult fat body enhancer of the Drosophila Adh gene (Falb and Maniatis $1992 \mathrm{~b}$. These two results imply that an active AEFl repressor that can bind to the aefl site is present in fat bodies but does not act at this site. 
The dsxA and bzip1 sites synergistically activate transcription in fat bodies

We used other site-specific mutations to determine the function of the two remaining binding sites in o. Both the triple mutation, which reduces binding to all three sites, and a double mutation, which leaves only the bzipl site intact, produce no detectable activation in fat bodies [Fig. 2f, $\left.\mathrm{d}_{;}\left(\mathrm{a}^{-} \mathrm{d}^{-1} \mathrm{~b}^{-}\right)_{4},\left(\mathrm{a}^{-} \mathrm{d}^{-1} \mathrm{~b}^{+}\right)_{4}\right]$. Likewise, a double mutation leaving only the dsxA site intact does not activate in fat bodies [Fig. $2 \mathrm{e}\left(\left.\mathrm{a}^{-} \mathrm{d}^{+} \mathrm{b}^{-}\right|_{4}\right)$. However, the 8-bp changes that restore both the dsxA and bzipl sites yield a tetramer that directs very strong expression, indistinguishable from that of the wild-type tetramer in female fat bodies (Fig. 2c). This striking and apparently all-or-none cooperative activation demonstrates strong synergism between proteins bound to the two sites.

The staining results also show that no expression occurs in male fat bodies when both dsxA and bzipl sites are present. This suggests that the female-specific DSX ${ }^{\mathrm{F}}$ protein, but not the male-specific $\mathrm{DSX}^{\mathrm{M}}$, cooperates with proteins bound at bzipl to activate transcription.

\section{Ovarian transcription is activated by bzip 1 but not by $d s x A$}

The dsxA site is irrelevant to the strong ovarian expression observed when aefl repression is removed. The 4-bp changes that reduce in vitro binding of dsx proteins by $>25$-fold have no detectable effect on this ovarian expression [Fig. 2, d vs. $c\left(a^{-} d^{-1} b^{+}\right)_{4}$ vs. $\left(\left.a^{-} d^{+} b^{+}\right|_{4}\right]$. This strongly implies that $\mathrm{DSX}^{\mathrm{F}}$ protein is inactive in ovaries, as also implied by our RNase protection experiments (see below). In contrast, the bzip1 site is sufficient to activate ovarian transcription. This is suggested by the strong expression from $\left(\mathrm{a}^{-} \mathrm{d}^{-1} \mathrm{~b}^{+}\right)_{4}$ and confirmed by the observation that $b^{-}$is the only mutation of $o$ that decreases ovarian transcription [Fig. 2, c vs. e $\left(\mathrm{a}^{-} \overline{\mathrm{d}}^{+} \mathrm{b}^{+}\right)_{4}$ vs. $\left(\left.a^{-} d^{+} b^{-}\right|_{4}\right]$. The other relevant pairwise comparisons between mutant tetramers support these conclusions about dsxA and bzipl function in ovarian regulation (Fig. $2 b-f)$. These results show that a single bzipl site is sufficient to activate ovarian transcription and, unlike in fat bodies, synergism from dsxA is unnecessary. This suggests that a different activator protein binds to bzipl in ovaries or that the same protein at substantially higher concentration or activity is present in ovaries.

We observed above that the aefl site represses the ovarian expression directed by the entire o tetramer. Because dsxA is irrelevant to this expression, aefl must repress activation by bzipl. This conclusion is supported by the similarity between changes in ovarian staining intensity of mutated o tetramers and the ratio of aef $l$ and bzipl binding affinities in those mutations (Figs. 1 and 2): $\left(\left.a^{-} d^{+} b^{-}\right|_{4}<\left(a^{-} d^{-1} b^{-}\right)_{4}<\left(a^{-} d^{-1} b^{+}\right)_{4}\right.$. In this series the $a^{-} d^{-1}$ mutation reduces AEF1 binding below the level of the $a^{-}$mutation alone yet has no effect on DmC/EBP binding (Fig. 1 legend). In summary, ovarian expression is directed by the bzip 1 site, repressed by the aefl site, and unaffected by the dsxA site.
The DSX $X^{F}$ protein activates transcription from $d s X A$ in fat bodies

As shown above, the few base-pair changes that reduce DSX ${ }^{\mathrm{F}}$ binding to dsXA also abolish transcriptional activation by $\mathrm{o}$ in fat bodies. This strongly implicates $\mathrm{DSX}^{\mathrm{F}}$ in activating fat body transcription from a single site in o. To test this implication, we moved the $a^{-} d^{+} b^{+}$tetramer construct into chromosomally female flies that have mutant $d s x$ alleles. One of these alleles, $d s x^{M+R 15}$, is a deletion of the entire $d s x$ locus (Baker et al. 1991). The other, $\operatorname{In}(3 \mathrm{R}) d s x^{D+R 3}$, is an inversion with a breakpoint in the $d s x$ gene and produces a shortened transcript (Duncan and Kaufman 1975; R. Nagoshi, pers. comm.). Flies with both mutant alleles have an extreme intersexual phenotype, as judged by abdominal pigmentation, genital structure, and bristle patterns, indicating that $\mathrm{DSX}^{\mathrm{F}}$ activity is either eliminated or greatly reduced $(\mathrm{R}$. Nagoshi, pers. comm.).

If $\mathrm{DSX}^{\mathrm{F}}$ is involved in fat body activation by o, the $d s x$ mutations should abolish or substantially reduce transcription directed by the $\mathrm{a}^{-} \mathrm{d}^{+} \mathrm{b}^{+}$tetramer. In accord with this prediction, no fat body expression was observed in flies carrying both $d s x$ mutant alleles (Fig. 2b). In contrast, the same tetramer directed strong expression in female heterozygotes with one copy of the wild-type $d s x$ allele and one copy of either mutant allele (Fig. 2b). Unfortunately, the extreme intersexual phenotype of the double mutant includes a loss of ovaries. This prevents a test of the hypothesis that DSX ${ }^{\mathrm{F}}$ protein, like the dsxA site, is irrelevant to o-directed ovarian expression.

Our genetic and molecular data leave little doubt that $\mathrm{DSX}^{\mathrm{F}}$ activates transcription from the single dsXA site in the o enhancer element. Both the protein and its binding site are necessary for transcriptional activation in fat bodies.

\section{$D m C / E B P$ protein does not appear to activate from bzip1 in either fat bodies or ovaries}

Because the slbo gene encodes the DmC/EBP protein that binds bzipl in vitro, it is a candidate to positively regulate transcription from the bzipl site. Its ovarian expression further strengthens this possibility in ovaries (Rørth and Montell 1992).

To examine the possibility that slbo regulates through bzipl, we introduced the $\left(\mathrm{a}^{-} \mathrm{d}^{+} \mathrm{b}^{+}\right)_{4}$ construct into slbo mutant flies. Because null mutations of the slbo gene are recessive lethals, we used one null allele $\left(s l b o^{e 7 b}\right)$ together with $s l b o^{e 14 a}$, a hypomorphic allele that produces a moderate slow border cell migration phenotype when homozygous (Montell et al. 1992; D. Montell, pers. comm.). Fat body staining from the $\left(\mathrm{a}^{-} \mathrm{d}^{+} \mathrm{b}^{+}\right)_{4}$ construct was not different between flies with these two mutant alleles and those with wild-type slbo alleles /data not shown). This result is consistent with findings that the DmC/EBP transcript was undetectable in adult fat bodies (Rørth and Montell 1992; P. Rørth, pers. comm.). It suggests that either extremely low levels of slbo protein are capable of directing fat body expression that is much 
stronger than in ovaries or, more likely, that a different regulatory protein acts through bzipl in fat bodies.

There was also no reduction in ovarian expression. To increase the sensitivity of the bzipl site regulation to levels of slbo protein, we introduced the $\mathrm{b}^{-}$mutation of o $\left(\mathrm{a}^{-} \mathrm{d}^{+} \mathrm{b}^{-}\right)$into the same double mutant flies. If slbo regulates through bzipl, then reducing bzipl affinity for the DmC/EBP product of slbo should raise the threshold of $\mathrm{DmC} / \mathrm{EBP}$ concentration necessary for activation. No change in reporter expression was observed in either ovaries or fat bodies (data not shown). We conclude that slbo is unlikely to regulate through bzipl. Because bZIP proteins often occur as families with related DNA bindingspecificities, we hypothesize that another family member, or members, is responsible for activation through bzipl, perhaps binding this site more tightly than $\mathrm{DmC} /$ EBP.

\section{The binding of $D S X^{F}$ and $A E F 1$ proteins to $\underline{O}$ is mutually exclusive}

The overlapping binding sites for AEF1 and DSX ${ }^{\mathrm{F}}$ suggest that these repressor and activator proteins may bind competitively, perhaps providing an explanation for the different effects of the aef 1 site in fat bodies and ovaries. To investigate this possibility, we examined binding competition using gel shift assays, radiolabeled o DNA, and protein extracts from baculovirus or bacterial cells overexpressing $\mathrm{DSX}^{\mathrm{F}}$ or AEFl. As shown in Figure 3, when AEF1 concentration is held constant, increasing $\mathrm{DSX}^{\mathrm{F}}$ concentration diminishes binding by AEF1 and in-

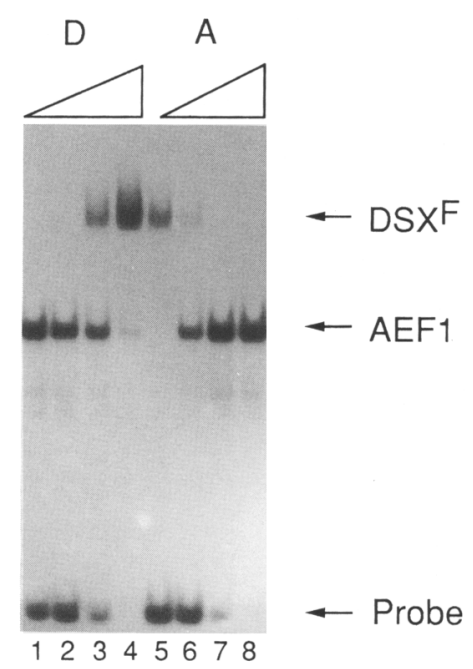

Figure 3. Binding competition between DSX ${ }^{F}$ and AEF1. The mobility shift assays shown in lanes $1-4$ result from binding reactions with increasing amount of baculovirus-expressed $\operatorname{DSX}^{\mathrm{F}}[(\mathrm{D}) 0,0.02,0.1,0.5 \mu \mathrm{l}$ of extract $]$ and a constant concentration of bacterially expressed AEFl $(0.05 \mu \mathrm{l}$ of extract $)$. Lanes 4-8 show reciprocal experiments with increasing AEFl concentration [(A) 0, 0.01, 0.1, 0.5 $\mu$ l of extract $)]$ and constant DSX ${ }^{\mathrm{F}}$ concentration $(0.05 \mu \mathrm{l}$ of extract). The identities of the bands are indicated by arrows and labels. creases binding by DSX ${ }^{\mathrm{F}}$. In reciprocal experiments, increasing AEFl concentration has the opposite effect. We conclude that the two proteins compete for binding to 0 in a mutually exclusive manner.

The ratio between $D S X^{F}$ and $A E F 1$ transcripts is extremely low in ovaries but high in fat bodies

A simple hypothesis to explain aefl activity and dsxA inactivity in ovarian regulation by o and the converse in fat bodies is that the ratio of the competitively binding AEFl and DSX ${ }^{\mathrm{F}}$ regulatory proteins is substantially different in the two organs. We examined this possibility with RNase protection experiments. Using a $\mathrm{DSX}^{\mathrm{F}}$-specific RNA probe, we observed the $\mathrm{DSX}^{\mathrm{F}}$ transcript in RNA preparations from whole female flies, females without ovaries, female abdominal cuticle, and females without ovaries or abdominal cuticle (Fig. 4A). The abdominal cuticle has an adhering layer of fat body cells on its inner surface and is generally accepted as the purest source of adult fat body tissue. In contrast to the abundance of $D_{S X^{F}}$ transcript in the other female samples, no $\mathrm{DSX}^{\mathrm{F}}$ transcript was detected in RNA preparations from ovaries or from male flies (Fig. 4A). When normalized by $\alpha 1$-tubulin mRNA signals in the same gel, the relative abundance of $\mathrm{DSX}^{\mathrm{F}}$ mRNA was, in decreasing order, abdomen, thorax, and ovaries. Prolonged autoradiographic exposure of the gel revealed no DSX ${ }^{\mathrm{F}}$ signal in the ovarian lane, demonstrating that this transcript is at least 100-fold lower in ovaries than abdomens.

Similar RNase protection experiments were done with a probe from the gene for AEFl (Fig. 4B). AEFl transcript was detected in RNA preparations from both whole males and females. In females, when normalized by the $\alpha$-tubulin mRNA signals (Kalfayan and Wensink 1982; Matthews et al. 1989), AEFl transcript in ovaries was two- to threefold more abundant than in the female fat body preparation and in females without ovaries.

We conclude that the ratio between AEF1 and DSX ${ }^{\mathrm{F}}$ transcripts is consistent with a competition model for the different regulatory effects of aefl and dsxA sites in ovaries and fat bodies. If the transcript levels reflect protein levels, then the AEFl protein can bind and repress in ovaries because it has little or no competition from $\mathrm{DSX}^{\mathrm{F}}$ protein. Conversely, because it is at substantially higher concentration in fat bodies, $\mathrm{DSX}^{\mathrm{F}}$ can bind, exclude the $A E F l$ repressor, and activate transcription in cooperation with a protein bound to the bzipl site.

A tetramer of the $r$ enhancer element is sufficient to activate nearly ubiquitous expression in both sexes

To examine the independent transcriptional activation specificities of $\underline{r}$, the other enhancer element of the $\underline{0-r}$ enhancer, we tested a tetramer of $\underline{r}$ either with or without a 5-bp substitution [Fig. 1, $\left.\underline{\mathrm{r}}_{4}(\underline{\mathrm{r}} \underline{\mathrm{sub}})_{4}\right)$. This substitution eliminates the refl site and all transcriptional synergism from $r$ in the normal o-r enhancer (An and Wensink 1995). In both tetramers, the first copy of $r$ is at the same position relative to the promoter of the reporter 

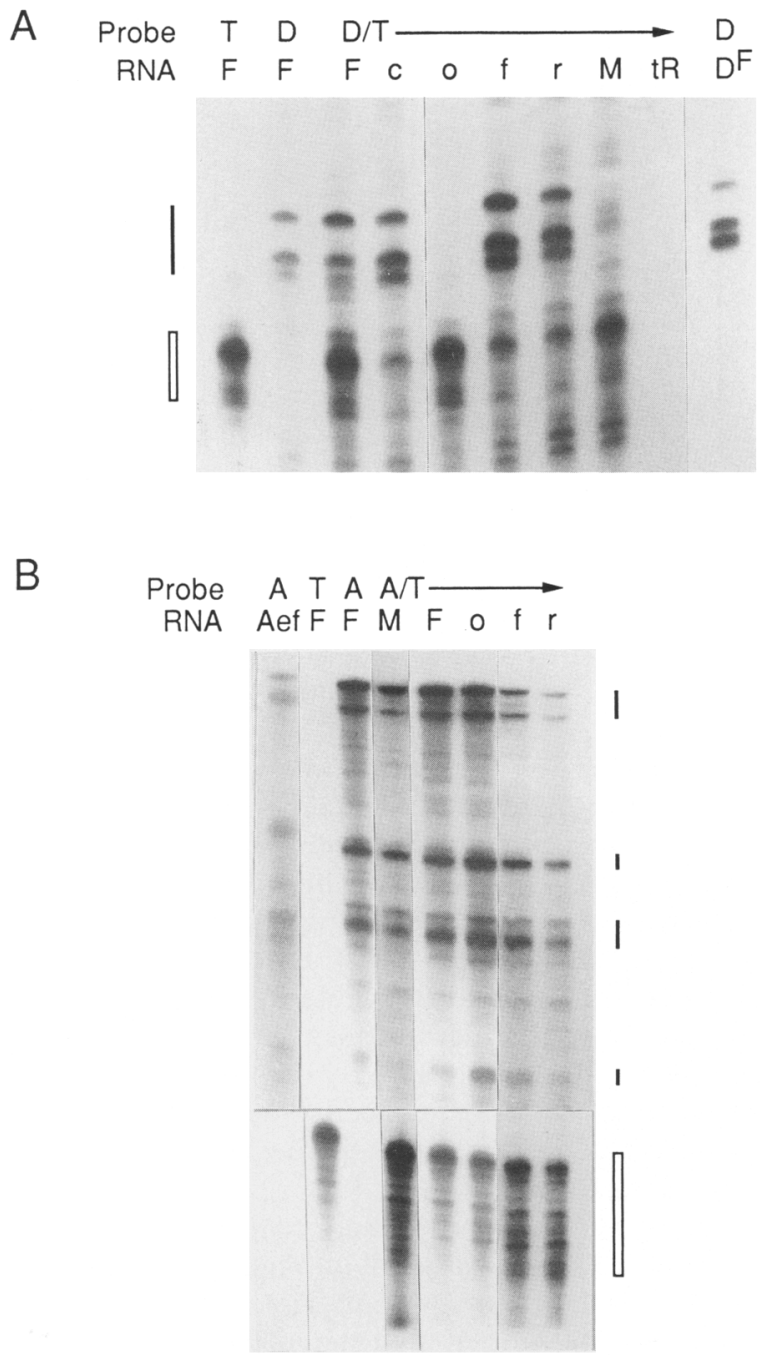

Figure 4. Tissue distributions of $D S X^{F}$ and AEF1 RNA. RNase protection assays using probes specific for $\operatorname{DSX}^{\mathrm{F}} \mathrm{mRNA}(A)$ and AEF 1 mRNA $(B)$. The positions of protected DSX ${ }^{\mathrm{F}}$ and AEF 1 signals are indicated by solid vertical bars and $\alpha 1$-tubulin signals by open bars. Total cellular RNA was from the following sources: (F) Female total; (c) female carcass (female without ovaries); (o) ovaries; (f) female fat body (abdominal cuticle and adhering fat body cells); (r) remaining parts (females without o and $\mathrm{f}_{;}(\mathrm{M})$ male total; $(\mathrm{tR})$ yeast $\mathrm{tRNA} ;\left(\mathrm{D}^{\mathrm{F}}\right)$ in vitro-transcribed

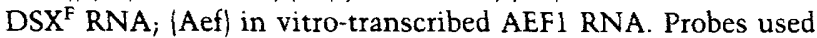
were ${ }^{32} \mathrm{P}$-labeled in vitro-transcribed RNA using portions of cDNA templates specific for $\alpha 1$-tubulin $(T), D_{S X}{ }^{F}(D)$, and AEF1 (A).

gene as it was in the fully active $\mathrm{FBE} /$ reporter construct (Coschigano and Wensink 1993). The other three copies were farther upstream.

Transgenic $\underline{r}_{4}$ flies had an unexpected staining pattern (Fig. 5a,b). Expression is very strong and nearly ubiquitous in both females and males. In both sexes, the spectrum of expression in major body parts includes but is not limited to mouth parts, Johnston's organ, maxillary palpus, head, thorax, legs, wing veins, and almost all internal organs of the abdomen and thorax. The eyes and alimentary canal do not appear to stain in either sex. The lack of staining in eyes is likely to be attributable to their resistance to dye penetration (O'Donnell et al. 1994). The only other organs not staining even after prolonged exposure to the staining buffer are the ovaries and testes where dye penetration is known to occur (Lis et al. 1983). Other male reproductive organs, such as accessory glands, anterior ejaculatory duct, and ejaculatory bulbs stain very intensely. The staining produced by $\underline{r}_{4}$ is attributable to the $\underline{r}$ enhancer element, because the $(\underline{\mathrm{r} \text { sub }})_{4}$ directs no detectable expression in any female tissue and only the ejaculatory bulbs and malpighian tubules stained in males, although at much reduced levels relative to the wild-type r tetramer (Fig. 5).

We conclude that $\underline{r}$, the 11 -bp refl-binding site, is an activator in all nongonadal tissue and, by itself, is not responsible for either the sex or fat body specificity of $\underline{o-r}$. However, in the context of the o-r enhancer it becomes an obligatory component in sex- and fat bodyspecific transcription.

\section{Discussion}

The o-r enhancer has four protein-binding sites that together direct sex- and tissue-specific transcription in Drosophila. A previous study identified these four sites and examined their in vivo activities in a single copy of the enhancer through use of site-specific mutations and germ-line transformation assays (An and Wensink 1995). That study led to the conclusion that one site was irrelevant, three were involved in tissue-specific activation, and one of those was also necessary for sex specificity. In this study we have assayed multimers of individual and combined sites to reveal potential combinatorial specificities and synergistic activities of each site. Results from these germ-line transformation assays and supplementary molecular/genetic studies lead us to propose a fairly simple hypothesis for regulation of sex specificity and a more complex one for the synergistic interactions that direct tissue specificity (Fig. 6).

The individual roles of the sites and their binding proteins can be summarized as follows. The bzipl and refl sites are the only two that can independently activate a promoter in our assay system. Furthermore, they must be multimerized to produce this activation. For example, four copies of refl stimulate a promoter in vivo, but one copy has no effect. As in previous cell transfection studies of the SV40 enhancer and other enhancers, this implies that more than one interaction with the promoter must take place for transcription to be activated (Ondek et al. 1988; Tjian and Maniatis 1994). This also is likely to be the mechanistic basis of synergy between single copies of bzipl and refl. These two sites offer an excellent example of combinatorial specification of the tissue in which transcription takes place, because individually, as multimers, the sites specify different tissue distributions of transcription (ovarian somatic tissue by bzipl and all nongonadal tissues by refl; Figs. 2 and 5), whereas 


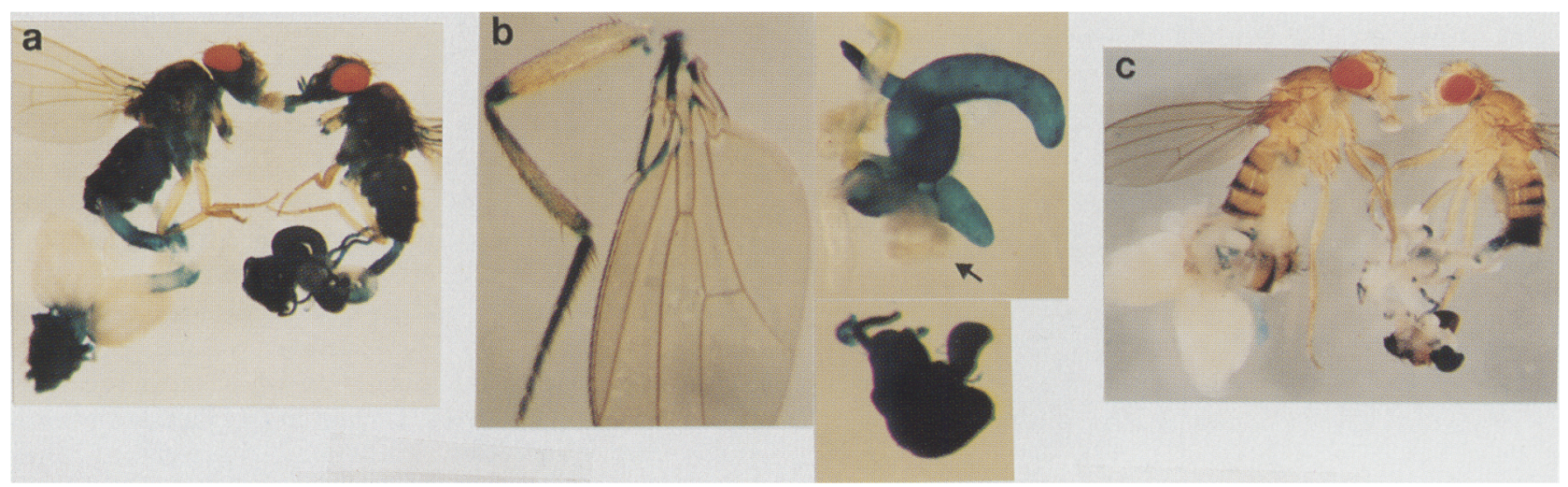

Figure 5. $\beta$-Galactosidase activity distribution in flies carrying $\underline{r}_{4}(a)$ and $(\underline{r} \text { sub })_{4}|c| .(b)$ Dissected body parts from $\underline{r}_{4}$ flies. (Clockwise) Stained wing and leg; part of male reproductive organs with stained accessory glands, ejaculatory duct, but unstained testes (arrow); and heavily stained ejaculatory bulbs.

together they specify still another tissue distribution [fat bodies only (An and Wensink 1995)].

The other two sites, aefl and dsxA, do not activate transcription independently in our assay but, instead, modify the activity of bzipl (Fig. 6). They may also modify the activity of refl, but we have no evidence for this and adopt the simplest working hypothesis that they only modify activity of the bzipl site which they both overlap. We find that the aefl site plays a role in tissue specificity by repressing ectopic transcription in ovaries that can be directed by bzip1. This repression may be particularly important in the context of the entire regulatory region of $Y p$ genes where several bzip sites occur in a spacing similar to that in the bzipl tetramer (An and Wensink 1995). The dsxA site, on the other hand, plays two roles in specificity. First, it is responsible for all of the sex specificity of the o-r enhancer. In this study we show that the DSX ${ }^{\mathrm{F}}$ protein is responsible for the activation by the dsxA site in females and the overwhelming evidence from this and previous work indicates that $\mathrm{DSX}^{\mathrm{M}}$ is responsible for the repression by dsxA in males (Coschigano and Wensink 1993). Finally, the DSX ${ }^{\mathrm{F}}$ protein also plays a role in tissue specificity by eliminating repression by aef1 in fat bodies. Our evidence indicates that this antirepression by DSX ${ }^{\mathrm{F}}$ occurs by competition between DSX ${ }^{\mathrm{F}}$ and AEFl for the overlapping dsxA and aef1 sites. This implies that the AEF1 protein may not only repress ectopic expression but may also modulate transcription levels in fat bodies.

\section{Proteins that activate from bzip1 and ref1}

DmC/EBP does not appear to be the bZIP protein that activates from bzipl in fat bodies or ovaries. Our genetic experiments showed no effect of the slbo gene on $\underline{0}_{4}$ directed expression in either organ. The implication that DmC/EBP is unlikely to act from bzipl in fat bodies is supported by the lack of detectable DmC/EBP transcripts in adult fat bodies (Rørth and Montell 1992; P. Rørth, pers. comm.). Support for the implied lack of
$\mathrm{DmC} / \mathrm{EBP}$ activity in ovaries is given by the difference between the expression patterns of DmC/EBP /centripetal cells, border cells, and micropyle (Montell et al. 1992)] and bzipl tetramers (germarium, micropyle, and all follicle cell types, including centripetal and border cells; Fig. 2). Although these observations are negative evidence, they give considerable weight to the hypothesis that DmC/EBP does not act at bzipl. Furthermore, because bzipl tetramer activity requires $\mathrm{DSX}{ }^{\mathrm{F}}$ in fat bodies but not in ovaries, we conclude that different transcriptional activators are likely to operate at bzipl in the two organs (Fig. 6).

The refl site is most likely to bind either a nearly ubiquitous factor or a family of regulatory proteins that together are nearly ubiquitous. The involvement of ubiquitous or widely distributed factors in tissue-specific regulation has been well documented (e.g., Weintraub et al. 1991; Nelsen and Sen 1992; Andrews et al. 1993; Igarashi et al. 1994).

\section{Competition between DSX and AEF1}

The aef 1 site does not function in fat bodies but represses expression in ovaries. This tissue-specific behavior of aefl is opposite to that of dsxA, which activates in fat bodies but not in ovaries. The complementary activity of the two sites is likely attributable to the competitive DNA binding between AEF1 and DSX ${ }^{F}$ and the apparently different abundance of the two proteins in the two tissues (Figs. 3 and 4). This competition is shown in Figure 6.

The observed competitive binding by DSX ${ }^{\mathrm{F}}$ and AEF1 also reconciles the discrepancy between the observations that aefl in the o-r enhancer shows no activity in fat bodies, whereas an AEF1-binding site in the Adh enhancer (AAE) represses in those same fat bodies (Falb and Maniatis 1992b; An and Wensink 1995). This is likely attributable to the presence of an overlapping dsx-binding site in $0-r$ but not in AAE. Thus, it appears that there are two regulatory roles for the AEF1 repressor. First, it 


\section{FAT BODIES}

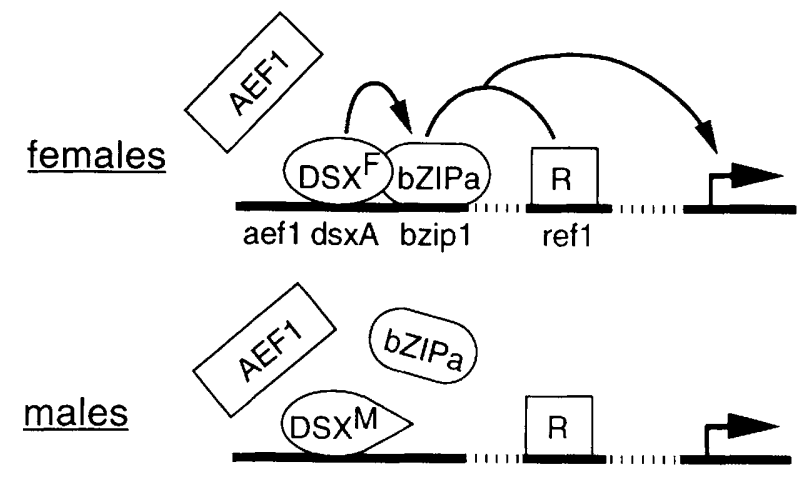

OVARIES

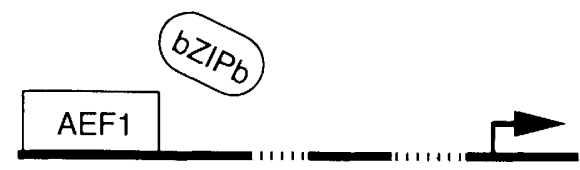

Figure 6. Model for transcriptional regulation by o-r. (Top) Activation in female fat bodies resulting from synergy among the $\mathrm{DSX}^{\mathrm{F}}$, $\mathrm{bZIPa}$, and $\mathrm{R}$ proteins. Curved arrows indicate activation, and the arc between bZIPa and R represents positive cooperativity. These three proteins are shown bound to their cognate sites on o-r DNA (solid lines). The AEFl repressor is shown unbound, excluded from its DNA site by DSX ${ }^{\mathrm{F}}$. Broken lines represent DNA between $\underline{0}, \underline{r}$, and the promoter (right-angle arrow). (Middle) Repression in male fat bodies resulting from DSX $^{M}$. DSX ${ }^{M}$ is shown bound to DNA, excluding the other two proteins from binding. (Bottom) Inactivation in ovaries and tissue specificity. The absence of dsx proteins allows AEFl to bind, excluding activators from the bzipl site. The bZIPa and bZIPb proteins activate from the bzipl site but have different tissue distributions.

appears to act upon AAE to reduce the levels of Adh in fat bodies. Second, it appears to regulate tissue specificity in $\mathrm{o}-\mathrm{r}$ by repressing ectopic activation in ovaries. Its role in ensuring fat body-specific expression by $0-r$ is in accord with many other examples of negative factors contributing to tissue-specificity (Goodbourn and Maniatis 1988; Pierce et al. 1991; Jiang and Levine 1993; Sawada et al. 1994).

\section{How is fat body tissue specificity directed by $0-r$ ?}

Results from this and previous studies indicate that a bZIP protein in fat bodies is most likely to be the major determinant of o-r fat body specificity. We arrive at this conclusion primarily by a process of elimination. First, the aefl site and AEFl protein appear to play a role in preventing ectopic transcription and, perhaps, in negative modulation of transcription in fat bodies, but to play no role in tissue specific activation (Fig. 2; An and Wensink 1995). Second, the dsxA site and DSX ${ }^{\mathrm{F}}$ protein are dispensable because fat body-specific activation persists when $\mathrm{DSX}^{\mathrm{F}}$ is absent, for example, in males or dsx ${ }^{-}$ intersex flies (Coschigano and Wensink 1993; An and Wensink 1995). Third, the refl site seems unlikely to direct tissue specificity because a tetramer of it directs nearly ubiquitous expression (Fig. 3). The simplest interpretation is that refl binds a nearly ubiquitous protein that is able to activate in almost any tissue.

The simplest hypothesis for tissue specificity is that bzipl is the fundamental determinant of fat body-specific transcription, although the protein bound to this site does require either the refl-binding protein or $\mathrm{DSX}^{\mathrm{F}}$ for this specific activation.

\section{dsx proteins direct sex-specific transcription}

The sex specificity of o-r depends only on dsx protein action at dsxA. In part, this is shown by the negative evidence that all other regulatory sites in $0-r$ act without sex specificity and by the positive evidence that base changes that specifically effect binding by dsx protein are also essential for any expression to be directed in females by $0-r$ (Figs. 1, 2, and 5). Furthermore, our genetic experiments showed that DSX ${ }^{F}$ regulates $\mathrm{o}-\mathrm{r}$ through the dsxA site (Fig. $2 b$ ) and both DSX ${ }^{F}$ and $\overline{D S X}^{M}$ regulate sex specificity through 27 bp of DNA that includes the dsxA site (Coschigano and Wensink 1993).

We propose the model shown in Figure 6 to account for sex-specific regulation in fat bodies. In males, the activator $\mathrm{DSX}^{\mathrm{F}}$ is missing, thereby eliminating its positive effect on the adjacent bZIP protein. Furthermore, DSX ${ }^{M}$ is present and represses bZIP activity by steric hindrance from its bulkier male-specific carboxy-terminal domain. Alternatively, the male specific domain may inactivate the bound bZIP protein (not shown in Fig. 6). Eliminating bZIP activity eliminates the necessary synergy with an activator at the refl site and therefore yields no expression. AEFl is unlikely to repress in male fat bodies through $0-r$ because of steric hindrance from DSX ${ }^{M}$.

In female fat bodies, there is synergism between DSX ${ }^{\mathrm{F}}$ and bZIP activator either through cooperative binding or by altering bZIP activity after that activator is bound. As an example of the latter, DSX ${ }^{\mathrm{F}}$ may help bZIP form a stable open complex at the promoter, as suggested for the case of NF-Y interaction with C/EBP $\alpha$ (Milos and Zaret 1992). The ability of bZIP proteins to interact with other transcriptional factors has been well documented (for review, see Nolan 1994). As shown in Figure 6, the second positive transcriptional effect of $\mathrm{DSX}^{\mathrm{F}}$ in female fat bodies is to interfere with binding and repression by AEF1.

This model predicts the effect of dsx proteins in two different types of intersex flies. In one type, both $D S X^{F}$ and $\mathrm{DSX}^{\mathrm{M}}$ are present, producing an equilibrium between activation by DSX ${ }^{\mathrm{F}}$ and repression by $\mathrm{DSX}^{\mathrm{M}}$ (Fig. 6). In the other type, both DSX ${ }^{\mathrm{F}}$ and $\mathrm{DSX}^{\mathrm{M}}$ are absent, allowing bZIP and AEF1 to have their conflicting effects. In either case, the model predicts transcription levels between those of wild-type females and males. This prediction agrees with observed expression levels of $Y p$ genes and the $Y p$ fat body enhancer/reporter genes in 
intersexual flies (Bownes and Nöthiger 1981; Bownes et al. 1990; Coschigano and Wensink 1993).

\section{Indirect and direct regulation of sex specificity by $d s x$ proteins}

Sex-specific regulation of the o-r enhancer is different in fat bodies and ovaries. First, the dsxA site is irrelevant in ovaries but essential in fat bodies. Second, the dsx female protein that regulates at dsxA has no detectable transcripts in ovaries but moderately abundant transcripts in fat bodies. We conclude that dsx proteins regulate $\underline{\underline{O}-\mathrm{r}}$ directly in fat bodies and indirectly in ovaries. Indirect regulation of ovarian expression must occur at some level because ovaries do not form without $d s \times$ activity (Fig. 2j; Baker and Ridge 1980). In contrast, in fat bodies, where direct regulation of $\underline{0-r}$ does occur, the tissue forms in $d s x$ mutants that have little or no dsx protein [Fig. 2j; (Baker and Ridge 1980)]. The conclusion that dsx proteins act directly in fat bodies and indirectly in ovaries provides an explanation for a previous finding. Nonpermissive conditions in mature adults carrying a temperature-sensitive mutation in the sex differentiation pathway lead to reduced $Y p$ expression in fat bodies but not in ovaries (Bownes et al. 1990). Although there are many other target genes indirectly regulated by the sex differentiation pathway, the $Y p$ genes and the $\underline{0-r}$ enhancer are the only current examples of direct regulation (Wolfner 1988; Burtis 1993).

\section{Materials and methods}

\section{Construction of transgenes}

The o tetramer was made as follows (Sambrook et al. 1989). The w3 region and the 4 bp immediately upstream of it in pUC/o$\overline{\mathrm{w} 3}$ (An and Wensink 1995) were substituted with the sequence TCTCCCCGATATCCCACCGTTCGAGC, yielding pUC/o. A DNA fragment of o flanked by SacII and SpeI sites was synthesized by a polymerase chain reaction (PCR) using $\mathrm{pUC} / \mathrm{O}$ as template, and primers TCCCCGCGGATATCGACGTGC and GACTAGTGACCTCGGCGG. This fragment was digested with SacII and SpeI and inserted into the SacII-SpeI site of pUC/ $\underline{0}$, yielding $\mathrm{pUC} / \mathrm{o}_{2}$. A Sall-Sall fragment containing two headto-tail copies of 0 was excised from the $\mathrm{pUC} / \mathrm{o}_{2}$, filled in with Klenow, and inserted into the HincII site of pUC19. A XbaISpeI fragment containing the $\underline{O}_{2}$ was transferred from this plasmid into the SpeI site of $\mathrm{pUC} / \underline{o}_{2}$, yielding $\mathrm{pUC} / \underline{\mathrm{o}}_{4}$ with headto-tail arrangement of monomers. Mutant versions of $\mathrm{pUC/ \underline {O } _ { 4 }}$ were constructed in the same way except using the o mutants (An and Wensink 1995).

The constructs for germ-line transformation were made by inserting Sall-Sall fragments from the $\mathrm{pUC} / \underline{\mathrm{o}}_{4}$ constructs into the Sall site of the hsp 70 promoter/lacZ reporter vector $/ \mathrm{Co}^{-}$ schigano and Wensink 1993). The first copies of o in $\mathrm{pUC} / \underline{\mathrm{o}}_{4} \mathrm{~s}$ are at the same position relative to the $h s p 70$ promoter as in $\mathrm{pUC} / \mathrm{o}-\mathrm{w} 3$. The sequences of mutations and positions of insertions of all constructs were verified by sequencing using the PCR sequencing kit from Promega.

The $\underline{r}$ tetramer was constructed as follows. Regions flanking $\underline{r}$ were modified by adding appropriate restriction sites with a PCR reaction using the o-w3 carrying the sub 5 substitution $\mid A n$ and Wensink 1995) as template and primers: GACTAGTCT-
TAAAATAATCAGG and CCGCTCGAGTCTAGTGGATC. Then, a SpeI-Xbal fragment including $\mathrm{r}$ and some spacer DNA was excised, ligated in the presence of both SpeI and XbaI enzymes, and the product was used to replace the single-copy Spel-XbaI fragment, producing the tetramer. The $|\underline{\mathrm{r} \text { sub }}|_{4}$ was constructed in the same way except that the sub $\overline{3\{\mathrm{~A}}$ and Wensink 1995/ was introduced with the primer GACTAGTCTTAAAATACCGTCGCGTAGAGT in PCR. The SpeI-Xbal fragment from each tetramer were inserted into the $X b a I$ site of the $h s p 70$ promoter/lacZ reporter, yielding $\underline{r}_{4}$ tetramers, with the first copy of $\underline{r}$ at the same position relative to the $h s p 70$ promoter as in the $\underline{0-r} /$ reporter construct (An and Wensink 1995). All constructs were verified by restriction mapping.

\section{Germ-line transformation}

P-element-mediated germ-line transformation using D. melanogaster $r y^{506}$ strain were performed according to the methods of Spradling and Rubin (1982) and the modifications described in Coschigano and Wensink (1993), except that only heterozygous transformants were maintained and examined. The number of insertions in each transformed fly was determined by Southern blots. Only results from single insertion lines are reported.

\section{Histochemical assays of $\beta$-galactosidase activity}

The X-gal (5-bromo-4-chloro-3-indolyl- $\beta$-galactopyranoside) histochemical staining assay of $\beta$-galactosidase activity was done as described in Coschigano and Wensink (1993), except that heterozygous transformants were used and flies were dissected in Ringer's solution. At least five independently transformed lines were examined for each construct to account for any potential chromosomal position effects on expression.

\section{Competitive gel shift}

Approximately $0.1 \mathrm{ng}$ of ${ }^{32}$-end-labeled BamHI-SpeI fragment from $\mathrm{pUC} / \mathrm{o}$ was used as binding substrate in each gel shift reaction, incubated with various amount of bacterially expressed AEF1 and baculovinus-expressed DSX extracts at $4^{\circ} \mathrm{C}$ for $20 \mathrm{~min}$ in $20 \mu \mathrm{l}$ of buffer that was $25 \mathrm{~mm}$ HEPES (pH 7.6), 100 $\mathrm{mm} \mathrm{NaCl}, 0.1 \mathrm{~mm}$ EDTA, $1 \mathrm{~mm} \mathrm{DTT}, 2 \mu \mathrm{g}$ of poly $[\mathrm{d}(\mathrm{I}-\mathrm{C})] /$ poly $[\mathrm{d}(\mathrm{I}-\mathrm{C})], 100 \mu \mathrm{g} / \mathrm{ml}$ of BSA, and $10 \%$ glycerol. All reactions were fractionated on a $4 \%$ nondenaturing polyacrylamide gel $(0.5 \times$ TBE, containing $5 \%$ glycerol) that was prerun and run at $4^{\circ} \mathrm{C}$. The DSX $\mathrm{X}^{\mathrm{C}}$ extract and the recipe of the binding buffer were generous gifts from S. Cho (Brandeis University, Waltham, $\mathrm{MA}$ ); the AEF1 extract was a generous gift from D. Falb, T. Abel, and T. Maniatis (Harvard University, Cambridge, MA).

\section{RNase protection}

Female and male flies, 0 to 2 days old, were yeasted for 3 days before being anesthetized and dissected. Female flies were first dissected into two parts: ovaries and carcasses, defined as females without ovaries. The carcass was separated further into two parts: abdominal wall, and all remaining parts of the fly. Total cellular RNA was extracted from whole female and male flies, and from dissected body parts (Burtis and Baker 1989).

The RNase protection experiments were performed according to standard procedures (Sambrook et al. 1989). Approximately $60 \mu \mathrm{g}$ of total RNA was used for each reaction. The DSX mRNA-specific probe spans from 95 nucleotides $5^{\prime}$ to $105 \mathrm{nu}$ cleotides $3^{\prime}$ of the downstream splicing site of the third intron of dsx primary transcript. This probe and the $\alpha \mathrm{l}$-tubulin specific 
probe were generous gifts from Ming Tian (Harvard University, Cambridge, MAl.

\section{dsx and slbo mutant flies}

Transgenic lines with the $\left(a^{-} d^{+} b^{+}\right)_{4}$ or $\left(a^{-} d^{+} b^{-}\right)_{4}$ reporter fusions inserted into the $\mathrm{X}$ chromosome were crossed with appropriate $d s x$ and slbo alleles to generate the desired progeny. The balancer strains involved were TM2, $U b x, r y / M K R S, s b, r y$, and $\mathrm{Cyo} / \mathrm{Sco}$; ry/ry.

The parental $d s x$ strains /generously provided by R. Nagoshi, University of Iowa, Iowa City/ were $B^{S} \mathrm{Y} /+;+1+; d s X^{M+R 15} /$ TM6b $(T b, H u, e)$ and $B^{S} \mathrm{Y} /+;+1+; \operatorname{In}(3 \mathrm{R}) d s x^{D+R 3} e / \mathrm{TM} 6 \mathrm{~b}$ $(T b, H u, e)$. The genotype of assayed $d s x$ mutant female flies was * $/+;+1+; d s x^{M+R 15} / \operatorname{In}(3 \mathrm{R}) d s x^{D+R 3} e$, with * denoting the transgenes.

The parental slbo strain /generously provided by D. Montell, The Johns Hopkins University School of Medicine, Baltimore, $\mathrm{MD}$ ) was $\mathrm{cn} 1, \mathrm{slbo} \mathrm{o}^{\mathrm{e} b} / \mathrm{cn} 2$, Cyo; ry/ry, a deletion covering the slbo locus, and $c n 1$, slbo ${ }^{e 14 a} / \mathrm{cn} 2$, Cyo; ry/ry, an expression mutant (Montell et al. 1992; D. Montell, pers. comm.). The genotype of assayed slbo mutant female flies was $*+; c n 1, s l b o^{e 7 b} /$ cn1, slbo ${ }^{e 14 a} ; r y / r y$.

\section{Acknowledgments}

We gratefully thank Dean Falb, Ted Abel, Tom Maniatis, Pernile Rørth, Denise Montell, Sayeon Cho, Ming Tian, and Rod Nagoshi for advice and for generous gifts of proteins, RNA hybridization probes, or mutant fly strains. We also thank Marie Lossky, Sayeon Cho, Robert Kolouch, and Yue Wang for helpful criticism of the manuscript. This research was supported by U.S Public Health Service grants from the National Institutes of Health (GM21626 and GM46327).

The publication costs of this article were defrayed in part by payment of page charges. This article must therefore be hereby marked "advertisement" in accordance with 18 USC section 1734 solely to indicate this fact.

\section{References}

An, W. and P.C. Wensink. 1995. Three protein binding sites form an enhancer that directs the sex and fat body specific transcription of Drosophila yolk protein genes. EMBO /. (in press).

Andrews, N.C., K.J. Kotkow, P.A. Ney, H. Erdjument-Bromage, P. Tempst, and S.H. Orkin. 1993. The ubiquitous subunit of erythroid transcription factor NF-E2 is a small basic-leucine zipper protein related to the v-maf oncogene. Proc. Natl. Acad. Sci. 90: 11488-11492.

Baker, B.S. and K.A. Ridge. 1980. Sex and the single cell. I. On the action of major loci affecting sex determination in Drosophila melanogaster. Genetics 94: 383-423.

Baker, B.S., G. Hoff, T.C. Kaufman, M.F. Wolfner, and T. Hazelrigg. 1991. The doublesex locus of Drosophila melanogaster and its flanking regions: a cytogenetic analysis. $G e-$ netics 127: 125-138.

Birkenmeier, E.H., B. Gwynn, S. Howard, J. Jerry, J.I. Gordon, W.H. Landschulz, and S.L. McKnight. 1989. Tissue specific expression, developmental regulation and genetic mapping of the gene encoding C/EBP. Genes \& Dev. 3: 1146-1156.

Bownes, M. and R. Nöthiger. 1981. Sex-determining genes and vitellogenin synthesis in Drosophila melanogaster. Mol. \& Gen. Genet. 182: 222-228.

Bownes, M., M. Steinmann-Zwicky, and R. Nöthiger. 1990. Dif- ferential control of yolk protein gene expression in fat bodies and gonads by the sex-determining gene tra-2 of Drosophila. EMBO /. 9: 3975-3980.

Burtis, K.C. 1993. The regulation of sex determination and sexually dimorphic differentiation in Drosophila. Curr. Opin. Cell. Biol. 5: 1006-1014.

Burtis, K.C. and B.S. Baker. 1989. Drosophila doublesex gene controls somatic sexual differentiation by producing alternatively spliced mRNAs encoding related sex-specific polypeptides. Cell 56: 997-1010.

Burtis, K.C. and M.F. Wolfner. 1992. The view from the bottom: Sex-specific traits and their control in Drosophila. Semin. Dev. Biol. 3: 331-340.

Coschigano, K.T. and P.C. Wensink. 1993. Sex-specific transcriptional regulation by the male and female doublesex proteins of Drosophila. Genes \& Dev. 7: 42-54.

De Simone, V. and R. Cortese. 1991. Transcriptional regulation of liver-specific gene expression. Curr. Opin. Cell. Biol. 3: 960-965.

Duncan, I.W. and T.C. Kaufman. 1975. Cytogenetic analysis of chromosome 3 in Drosophila melanogaster: Mapping of the proximal portions of the right arm. Genetics 80: $733-752$.

Erdman, S.E. and K.C. Burtis. 1993. The Drosophila doublesex proteins share a novel zinc finger related DNA binding domain. EMBO I. 12: 527-535.

Falb, D. and T. Maniatis. 1992a. A conserved regulatory unit implicated in tissue-specific gene expression in Drosophila and man. Genes \& Dev. 6: 454-465.

- $1992 \mathrm{~b}$. Drosophila transcriptional repressor protein that binds specifically to negative control elements in fat body enhancers. Mol. Cell. Biol. 12: 4093-4103.

Goodbourn, S. and T. Maniatis. 1988. Overlapping positive and negative regulatory domains of the human $\beta$-interferon gene. Proc. Natl. Acad. Sci. 85: 1447-1451.

Igarashi, K., K. Kataoka, K. Itoh, N. Hayashi, M. Nishizawa, and M. Yamamato. 1994. Regulation of transcription by dimerization of erythoid factor NE-E2 p45 with small Maf proteins. Nature 367: 568-572.

Jiang, J. and M. Levine. 1993. Binding affinities and cooperative interactions with bHLH activators delimit threshold responses to the dorsal gradient morphogen. Cell 72: 741-752.

Kalfayan, L. and P.C. Wensink. 1982. Developmental regulation of Drosophila $\alpha$-tubulin genes. Cell 29: 91-98.

Lawrence, P.A. 1992. The making of a fly. Blackwell Scientific Publications, Oxford, UK.

Lis, J.T., J.A. Simon, and C.A. Sutton. 1983. New heat shock puffs and $\beta$-galactosidase activity resulting from transformation of Drosophila with an hsp70-lacZ hybrid gene. Cell 35: 403-410.

Matthews, K.A., D.F.B. Miller, and T.C. Kaufman. 1989. Developmental distribution of RNA and protein products of the Drosophila $\alpha$-tubulin gene family. Dev. Biol. 132: 45-61.

McKeown, M. 1992. Sex differentiation: the role of alternative splicing. Curr. Opin. Genet. Dev. 2: 299-303.

Milos, P.M. and K.S. Zaret. 1992. A ubiquitous factor is required for C/EBP-related proteins to form stable transcription complexes on an albumin promoter segment in vitro. Genes \& Dev. 6: 991-1004.

Montell, D.J., P. Rorth, and A.C. Spradling. 1992. Slow border cells, a locus required for a developmentally regulated cell migration during oogenesis, encodes Drosophila C/EBP. Cell 71: 51-62.

Nelsen, B. and R. Sen. 1992. Regulation of immunoglobulin gene transcription. Int. Rev. Cytol. 133: 121-149.

Nolan, G.P. 1994. NF-AT-AP1 and Rel-bZIP: Hybrid vigor and binding under the influence. Cell 77: 795-798. 
O'Donnell, K.H., C.-T. Chen, and P.C. Wensink. 1994. Insulating DNA directs ubiquitous transcription of the Drosophila melanogaster $\alpha 1$-tubulin gene. Mol. Cell. Biol. 14: 63986408.

Ondek, B., L. Gloss, and W. Herr. 1988. The SV40 enhancer contains two distinct levels of organization. Nature 333: 40 45.

Orkin, S.H. 1990. Globin gene regulation and switching: Circa 1990. Cell 63: 665-672.

Pierce, J.W., A.M. Fifford, and D. Baltimore. 1991. Silencing of the expression of the immunoglobulin kappa gene in non-B cells. Mol. Cell. Biol. 11: 1431-1437.

Rørth, P. and D.J. Montell. 1992. Drosophila C/EBP: A tissuespecific DNA-binding protein required for embryonic development. Genes \& Dev. 6: 2299-2311.

Sambrook, J., E.F. Fritsch, and T. Maniatis. 1989. Molecular cloning: A laboratory manual. Cold Spring Harbor Laboratory Press, Cold Spring Harbor, New York.

Sawada, S., J.D. Scarborough, N. Killeen, and D.R. Littman. 1994. A lineage-specific transcriptional silencer regulates CD4 gene expression during $T$ lymphocyte development. Cell 77: 917-929.

Small, S., A. Blair, and M. Levine. 1992. Regulation of evenskipped stripe 2 in the Drosophila embryo. EMBO $I$. 11: 4047-4057.

Spradling, A. and G.M. Rubin. 1982. Transposition of cloned P elements into Drosophila germ line chromosomes. Science 218: 341-347.

Struhl, K. 1991. Mechanisms for diversity of gene expression patterns. Neuron 7: 177-181.

Tjian, R. and T. Maniatis. 1994. Transcriptional activation: A complex puzzle with few easy pieces. Cell 77: 5-8.

Weintraub, H., R. Davis, S. Tapscott, M. Thayer, M. Krause, R. Benezra, T.K. Blackwell, D. Turner, R. Rupp, S. Hollenberg, Y. Zhuang, and A. Lassar. 1991. The myoD gene family: Nodal point during specification of the muscle cell lineage. Science 251: 761-766.

Wolfner, M.F. 1988. Sex-specific gene expression in somatic tissues of Drosophila melanogaster. Trends Genet. 4: 333-337. 


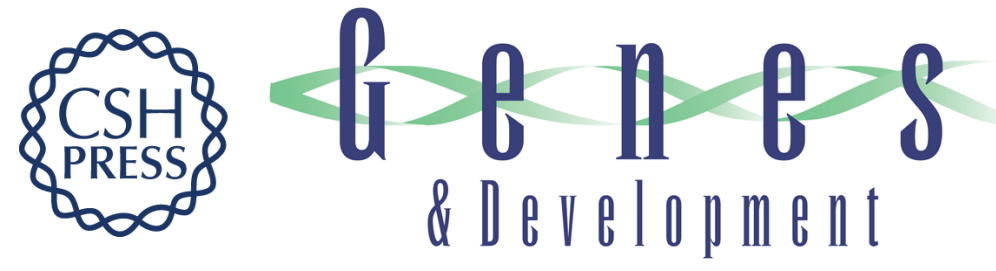

\section{Integrating sex- and tissue-specific regulation within a single Drosophila enhancer.}

W An and P C Wensink

Genes Dev. 1995, 9:

Access the most recent version at doi:10.1101/gad.9.2.256

References This article cites 38 articles, 15 of which can be accessed free at:

http://genesdev.cshlp.org/content/9/2/256.full.html\#ref-list-1

License

Email Alerting

Service

Receive free email alerts when new articles cite this article - sign up in the box at the top right corner of the article or click here.

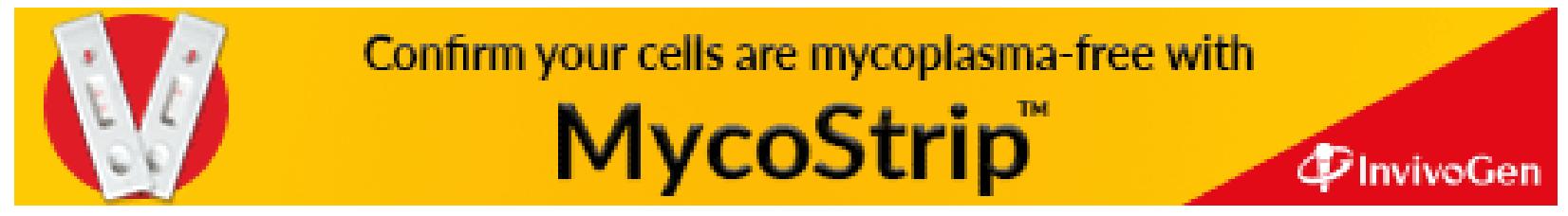

